\title{
Correlation Analysis between Traditional Chinese Medicine Syndromes and Gastrointestinal Bleeding after Percutaneous Coronary Intervention
}

\author{
Chenhao Zhang $\mathbb{D}^{1},{ }^{1}$ Chaolian Huang, ${ }^{1}$ Mingming Wang, ${ }^{2}$ Xiaolin Kong, \\ Guannan Liu, ${ }^{1}$ and Jie Wang iD ${ }^{3}$ \\ ${ }^{1}$ Wangjing Hospital, China Academy of Chinese Medical Sciences, Beijing 100102, China \\ ${ }^{2}$ Beijing First Hospital of Integrated Chinese and Western Medicine, Beijing 100026, China \\ ${ }^{3}$ Guanganmen Hospital, China Academy of Chinese Medical Sciences, Beijing 100053, China \\ Correspondence should be addressed to Jie Wang; wangjie0103@126.com
}

Received 27 November 2017; Revised 22 February 2018; Accepted 8 March 2018; Published 31 May 2018

Academic Editor: Caigan Du

Copyright (C) 2018 Chenhao Zhang et al. This is an open access article distributed under the Creative Commons Attribution License, which permits unrestricted use, distribution, and reproduction in any medium, provided the original work is properly cited.

Objective. To explore the characters of traditional Chinese medicine (TCM) syndromes after percutaneous coronary intervention (PCI) and to provide syndrome study theoretical evidence for TCM differentiation treatment after PCI through retrospective study. Methods. Patients with coronary heart disease (CHD) who underwent PCI in Cardiovascular Intervention Center of Wangjing Hospital during Dec. 2012 to Dec. 2014 and met the inclusion criteria were enrolled. Retrospective study was then conducted based on patients' clinical document and angiography data to explore the distribution pattern of TCM syndromes. Results. 801 patients were recruited in the study. TCM syndromes in descending order of their incidence were Qi deficiency and blood stasis syndrome, heart blood stasis syndrome, Qi and Yin deficiency syndrome, phlegm and blood stasis syndrome, Qi stagnation and blood stasis syndrome, Yang asthenia syndrome, heart and kidney yin deficiency syndrome to cold congeal, and blood stasis syndrome in a more to less order. Qi deficiency and blood stasis syndrome was in the most (occurring in 298 patients, 37.20\%); Qi and Yin deficiency syndrome occurred in 163 patients (20.35\%); heart blood stasis syndrome was shown in 126 patients (15.73\%); phlegm and blood stasis syndrome was shown in 95 patients (11.86\%). Conclusion. Qi deficiency and blood stasis syndrome was closely associated with post-PCI bleeding, implying that this syndrome might serve as a powerful predictor of GI bleeding as well as a potential supplement to the current predicting and scoring system of bleeding such as CRUSADE.

\section{Introduction}

With the development of modern medical technology, percutaneous coronary intervention (PCI), which is the major therapy of coronary heart disease (CHD), has helped countless patients. However, as the opening of diseased vessel, resolving of coronary occlusion or stenosis, some postoperative adverse event badly affects the long-term safety and efficacy, making it difficult for patient to get benefit in the long run. Gastrointestinal (GI) tract bleeding after PCI, which is one of the major adverse events and has intensively threatened patients' lives, is drawing more and more attention. In traditional Chinese medicine (TCM), CHD is categorized into "chest obstruction" or "heart pain," with the underlying etiology being "deficient origin and excessive superficiality." Although sufficient TCM studies have been conducted focusing on analysis of PCI-related TCM syndromes, investigations aiming to analyze postoperative adverse event, especially GI bleeding, are rare, which undoubtedly impeded the utility of TCM syndrome differentiation therapy in clinical practices.

The current retrospective study enrolled 801 patients undergoing PCI and analyzed the TCM syndrome characters of patients with GI bleeding or high bleeding risk and explored the correlation between GI bleeding and TCM syndromes as well, thus providing clinical evidence for the use of TCM to prevent GI bleeding after PCI in those with high bleeding risks. 


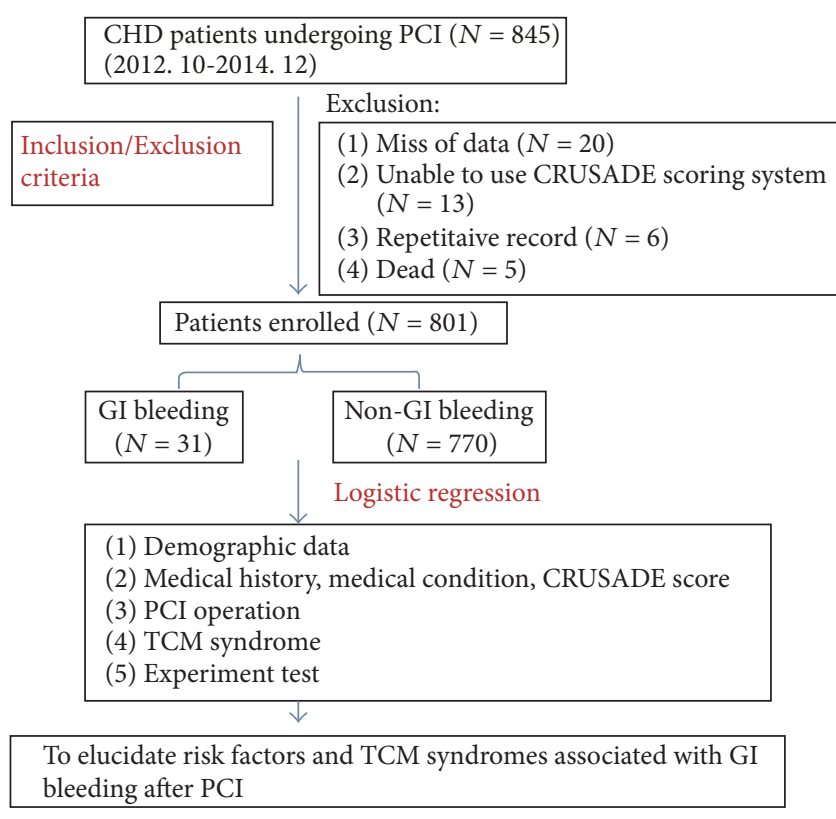

Figure 1: Flow chart.

\section{Materials and Methods}

2.1. Patients. 845 patients undergoing PCI in Cardiovascular Intervention Center of Wangjing Hospital during Oct. 2012 to Dec. 2014 were recruited, from which 44 participants who did not meet the including criteria were excluded, and 801 participants were enrolled in the end. 31 patients reported GI bleeding and 770 are without bleeding. Retrospective analysis was performed based on participants' original clinical documents and radiography data (as shown in Figure 1).

\subsection{Diagnostic Criteria}

2.2.1. Diagnostic Criteria for CHD. ST-elevated myocardial infarction (STEMI) was diagnosed according to the 2010 Guideline for the diagnosis and treatment of acute ST-elevated myocardial infarction published by Chinese Society of Cardiology [1]. Diagnosis of unstable angina pectoris (UAP) and non-ST-elevated myocardial infarction (NSTEMI) referred to 2012 Guideline for the diagnosis and treatment of non-ST-elevated myocardial infarction published by Chinese Society of Cardiology [2]. Stable angina pectoris (SAP) was diagnosed according to the 2007 Guideline for the diagnosis and treatment of chronic stable angina pectoris published by Cardiovascular Committee, China Medical Association [3].

2.2.2. Diagnostic Criteria for GI Bleeding after PCI. Recently significant hematemesis or melena and hrmatocrit (HCT) reduction by $\geq 15 \%$ occurred and/or hemoglobin decreased by $\geq 30 \mathrm{~g} / \mathrm{L}[3]$.

2.2.3. Criteria for Syndrome Differentiation. CHD was classified into the following eight syndromes: heart blood stasis syndrome, Qi deficiency and blood stasis syndrome, Qi stagnation and blood stasis syndrome, phlegm and blood stasis syndrome, cold congeal and blood stasis syndrome, Qi and Yin deficiency syndrome, heart and kidney yin deficiency syndrome, and Yang syndrome according to the differentiation standard for CHD angina pectoris from 〈Principles for Clinical Research of New Traditional Medicine〉 [4] revised by China Food and Drug Administration and the classification standard of chest obstruction from the national TCM higher education 12th five year version of textbook of 〈Internal Medicine of Traditional Chinese Medicine〉 [5].

\subsection{Inclusion Criteria}

(1) Meeting the criteria of $\mathrm{CHD}$, coronary angiography demonstrating stenosis $(>70 \%)$ in at least one vessel.

(2) At least one stent implantation during successful PCI operation and without operation related severe complication.

(3) Full and accurate clinical document and data.

\subsection{Exclusion Criteria}

(1) Severe illness in other systems, such as acute/chronic infection, blood disease, malignant neoplasm, and decompensated hepatocirrhosis.

(2) Active gastrointestinal ulcer or bleeding, stomach operation within three months, cerebral hemorrhage history, recent trauma surgery, and other affecting antithrombotic drug use disorders.

(3) With other severe organic cardiopathies, such as dilated cardiomyopathy, obstructive hypertrophy cardiomyopathy, and severe valvular diseases.

(4) Allergic constitution and cannot accept the drugs recommended by guideline.

(5) Incomplete clinical document or cannot comply with the follow-up and data collection process.

\subsection{Operation Rules and Definition}

(1) PCI Operation Rules. Coronary angiography (CAG) was conducted using SIMENS AXIOM ARTISDFC digital subtraction machine according to Judkins method. The regular puncture path was radial artery path, and PCI was operated according to 2005 American Heart Association Guideline for PCI. For patients without contradiction for antiplatelet agent, drug-eluting stent was recommended.

(2) Definition for Successful PCI Operation. CAG demonstrated significant dilation of target vessel, diameter of the rest stenosis $<20 \%$, and coronary blood flow return to TIMI3 [6].

2.6. Regulation of Drug Use during Perioperative Period. Enrolled patients planning to undergo PCI should take clopidogrel of loading dose (300 mg) (Plavix, Sanofi-Aventis) and chew an aspirin (300 mg) (Aspirin, Bayer) before operation. Heparin was administrated intravenously $80 \sim 100 \mathrm{IU} / \mathrm{kg}$ 
at the beginning of PCI operation; low molecular weight heparin or GPIIb/IIIa receptor antagonist (Tirofiban, Yuanda Pharmaceutical, Wuhan, China) was administered electively according to the decision of operator during operation. After the operation, Tirofiban was administrated intravenously and continuously for 36-48 hours; low molecular weight heparin was injected subcutaneously for 5-7 days; aspirin (100 mg) and clopidogrel $(75 \mathrm{mg}$ ) were taken orally; and the dose was determined by cardiologist. Antiplatelet or anticoagulation agent was withdrawn according to clinical conditions once severe bleeding occurred.

2.7. Regulations of Drug Use after Operation. For patients who underwent drug-eluting stent implantation, dual-antiplatelet therapy (DAPT) of aspirin $(100 \mathrm{mg} / \mathrm{d})$ and clopidogrel $(75 \mathrm{mg} / \mathrm{d})$ was required for at least one year. For those with bare-metal stent, DAPT was required for at least one month. Patients were prescribed with statins, ACEI, $\beta$ receptor blocker, and other drugs for secondary prevention of CHD after PCI. Risk factors including diabetes mellitus, hypertension, hyperlipidemia, and smoking were controlled.

2.8. CRUSADE Bleeding Score. ESC-NSTEACS recommended CRUSADE bleeding score [7] was referred to determine the classification from extremely low risk $(\leqslant 20)$, low risk $(21 \sim$ $30)$, moderate risk (31 40), high risk (41 50), and extremely high risk $(>50)$. Patients with CRUSADE score $\geqslant 41$ were determined as high risk of bleeding.

2.9. BARC Bleeding Definition. Bleeding was defined according to Bleeding Academic Research Consortium (BARC) classification referred by 2011 American Academic Research Consortium (ARC) [8].

2.10. Research Method. Based on patients' original clinical document and angiography data, participants with GI bleeding occurrence were assigned into patient group, while those without GI bleeding were control group. Retrospective analysis was conducted to compare the demographic characters and TCM syndrome features and to explore the correlation between GI bleeding after PCI and TCM syndrome.

\section{Clinical Data}

3.1. General Clinical Data. Participants' age, gender, height, weight, blood pressure, and heart rate were recorded.

3.2. Medical History and Current Symptoms. Previous medical history (hypertension, type 2 diabetes mellitus, lipid disorder, chronic heart dysfunction, chronic kidney dysfunction, anemia, previous cerebrovascular disease, and previous peptic ulcer), smoking history, admission diagnosis (STEMI, NSTEMI, UAP, and SAP), laboratory examination (serum creatinine, creatinine clearance rate, and hematocrit), heart failure symptom, and hospital stay (days) were recorded.

3.3. Operation Condition. Number of stent implantation and part of diseased vessel were recorded.
Participants' TCM symptoms, physical signs, tongue manifestation, and pulse were recorded.

CRUSADE score were evaluated.

3.4. Bleeding (Endpoint). The primary endpoint included incidence of GI bleeding, BARC score, and blood transfusion.

3.5. Data Collecting and Quality Control. In the current study, all data were obtained from the original clinical document, angiography information was exported from the catheter center of Wangjing Hospital, and clinical data acquisition was performed by physician or postgraduate of our department. Data was imported into EpiData database after check by researchers. TCM syndrome differentiation was first assessed by one experienced attending physician and confirmed by one at least associate chief physician at last. To guarantee the accuracy of data, each case was imported by at least two data administrators after consistency check and revision of any inconsistent data until the information was accurate. Two experienced physicians were designated as guide for quality control who were responsible for the supervision of data collecting process, data check, and data quality control. All information related to patients in the current study was well preserved and hided for the consideration of patients' privacy.

\section{Statistics}

4.1. General Principles and Methods. All statistical tests were performed using two-sided hypothesis tests at the level of $\alpha$ $=0.05$ ( $P \leq 0.05$ indicates statistical significance). Specific principles are as follows:

(1) Quantitative data was described with mean and standard deviation, and the hypothesis test was performed using $t$-test (normal distribution) or Wilcoxon ranksum test.

(2) Qualitative data was described with frequency and percentage, and the hypothesis test was performed using chi-square test or Fisher test. Rank data was analyzed using Wilcoxon rank-sum test.

4.2. Logistic Regression Model Was Applied to Analyze Risk Factors for GI Bleeding. Logistic regression model was built with GI bleeding being primary endpoint, demographic data, medical history, and TCM syndromes being covariant. The while process was performed in a 2-step manner: firstly, single factor logistic regression analysis was performed to screen out the significant variants $(\alpha=0.10)$. Thereafter, multiple factor regression was performed $(\alpha=0.05)$. Maximum likelihood algorithm was used for estimation; odd ratio (OR) and $95 \%$ CI were calculated.

Software SAS9.2 was applied for data analysis.

\section{Result}

5.1. Demographics Characteristics. 845 patients undergoing PCI from 1 Oct. 2012 to 31 Dec. 2014 were included into the current study, of which 20 patients were excluded due to miss 
of basic information, 13 patients were excluded due to the unavailability of CRUSADE score, 6 patients were doublerecorded, and 5 were dead. As a result, 801 participants were enrolled in the study finally with the average age of $64.03 \pm$ 12.12 years (youngest being 32 years while the oldest being 92 years), 531 males (66.29\%) and 270 females (33.71\%).

Medical History. 515 had hypertension (64.29\%), 296 had diabetes mellitus (36.95\%), 167 had lipid disorder (20.85\%), 85 had chronic heart dysfunction (10.61\%), 66 had chronic kidney dysfunction (8.24\%), 16 had anemia (2\%), 169 had cerebrovascular diseases $(21.1 \%), 63$ had peptic ulcer $(7.87 \%)$ and 365 had smoking history (45.56\%).

Admitting Diagnosis. 186 had acute STEMI (23.22), 153 acute NSTEMI (19.10\%), 342 unstable angina pectoris (42.70\%), and 120 stable angina pectoris (14.98\%). (Figure 2).

Distribution Pattern of TCM Syndromes. 126 patients presented with heart blood stasis syndrome (15.73\%), 298 were Qi deficiency and blood stasis syndrome (37.20\%), 47 Qi stagnation and blood stasis syndrome (5.87\%), 95 phlegm and blood stasis syndrome (11.86\%), 12 cold congeal and blood stasis syndrome (1.5\%), $163 \mathrm{Qi}$ and Yin deficiency syndrome $(20.35 \%), 28$ heart and kidney yin deficiency syndrome (3.5\%), and 32 Yang asthenia syndrome (4\%) (Figure 3). Table 1 shows figures about stent implantation and Table 2 shows figures about bleeding.

5.2. Clinical Characters of Patients Presenting with GI Bleeding after PCI. Among the total 801 patients enrolled in the study, GI bleeding occurred in 31 participants. Significant difference could be found between GI bleeding group and non-GI bleeding group in terms of age, gender, systolic pressure, serum creatinine, creatinine clearance rate, left anterior descending (LAD) branch being diseased vessel, and hospital stay $(P<0.05)$ (Table 3$)$.

In order to explore the correlation with GI bleeding, each predictor of CRUSADE score evaluation was analyzed. Significant difference could be found in of gender score, creatinine clearance rate score, systolic blood pressure score, CRUSADE 5 classification score, and CRUSADE 2 classification score when comparing the two groups $(P<0.05)$ (Table 4).

5.3. Correlation Analysis between TCM Syndromes with GI Bleeding. The distribution pattern of TCM syndromes in 31 GI bleeding participants: heart blood stasis syndrome (1), Qi deficiency and blood stasis syndrome (19), Qi stagnation and blood stasis syndrome (1), phlegm and blood stasis syndrome (1), cold congeal and blood stasis syndrome (1), Qi and Yin deficiency syndrome (3), heart and kidney yin deficiency syndrome (1), and Yang asthenia syndrome (4) (as shown in Figure 4). The results indicated significant difference in the distribution of Qi deficiency and blood stasis syndromes $(P<0.05)$ (as shown in Table 5).

Qi stagnation and blood stasis syndrome, heart blood stasis syndrome, phlegm and blood stasis syndrome, Qi

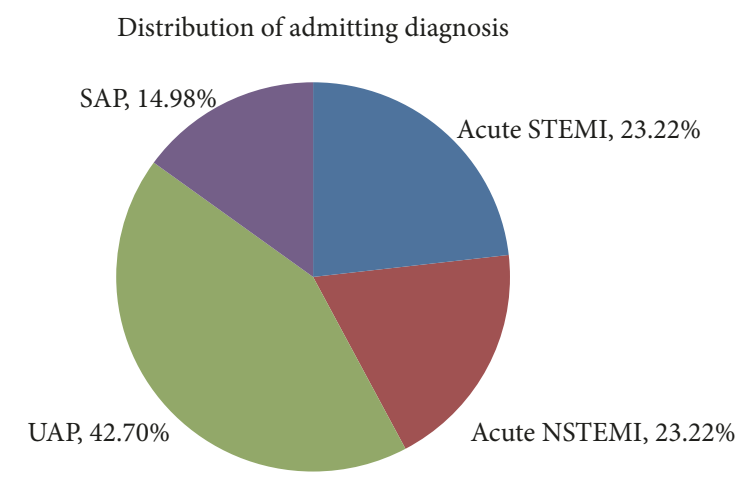

FIGURE 2: Distribution of admitting diagnosis.

TABLE 1: Figures about stent implantation.

\begin{tabular}{lc}
\hline & $N(\%)$ \\
\hline Diseased vessel & \\
Left anterior descending & $643(80.27)$ \\
Circumflex & $436(54.43)$ \\
Right coronary artery & $519(64.79)$ \\
Left main coronary artery & $70(8.74)$ \\
Others & $80(9.99)$ \\
Number of stent implantation & \\
1 & $289(36.08)$ \\
2 & $228(28.46)$ \\
3 & $127(15.86)$ \\
4 & $86(10.74)$ \\
$\geqslant 5$ & $71(8.86)$ \\
Average & $2.33 \pm 1.45$ \\
Total & 801 \\
\hline
\end{tabular}

TABLE 2: Figures about bleeding.

\begin{tabular}{lc}
\hline Indicator & $N(\%)$ \\
\hline BARC bleeding grade & \\
1 & $61(61.62)$ \\
2 & $25(25.25)$ \\
3 & \\
$3 \mathrm{a}$ & $8(8.08)$ \\
$3 \mathrm{~b}$ & $4(4.04)$ \\
3c & $1(1.01)$ \\
GI bleeding & $31(3.87)$ \\
Blood transfusion treatment after PCI & $7(0.87)$ \\
Bleeding site & \\
GI & $31(31.31)$ \\
Puncture site & $56(56.57)$ \\
Urinary tract & $2(2.02)$ \\
Retroperitoneal & $2(2.02)$ \\
Cerebral hemorrhage & $1(1.01)$ \\
Gums & $7(7.07)$ \\
\hline
\end{tabular}

and Yin deficiency syndrome, heart and kidney yin deficiency syndrome together with Yang asthenia were combined 


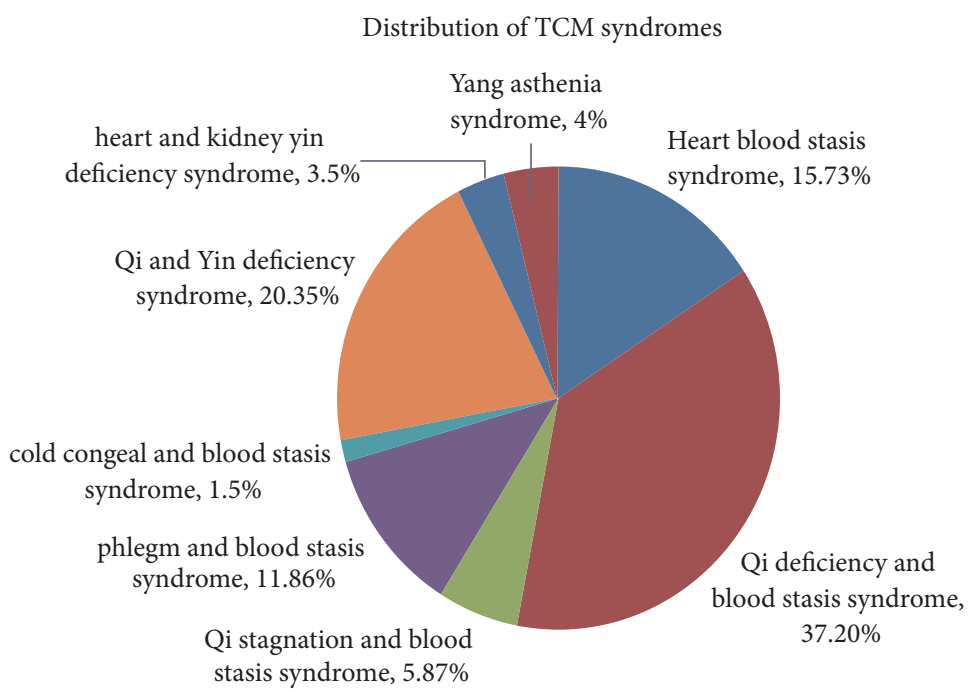

FIGURE 3: Distribution of TCM syndromes.

Distribution pattern of TCM syndromes in GI-bleeding participants

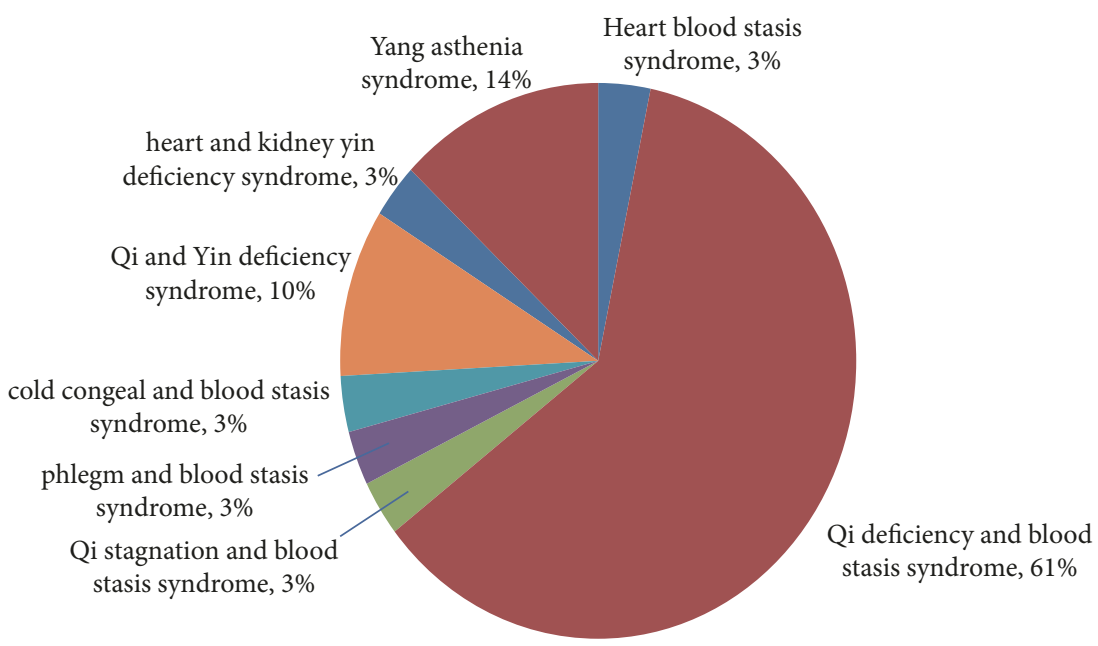

FIGURE 4: Distribution patterns of TCM syndromes in GI bleeding participants.

as "non-Qi deficiency and blood stasis syndrome." We compared the incidence of GI bleeding between Qi deficiency and blood stasis group $(n=19)$ and non-Qi deficiency and blood stasis group $(n=12)$ and found statistical significant difference $(P<0.05)$ (Table 6).

5.4. Single Factor Regression Analysis of Risk Factors and TCM Syndromes of GI Bleeding after PCI. Single factor logistic regression model was applied to screen the risk factors and TCM syndromes of GI bleeding after PCI. To fit the regression model properly and explain the result accurately, indicators of age, BMI, and number of stent implantation were transferred into categorical data. The results indicated that indicators of age, weight, gender, CRUSADE score (5 classifications), CRUSADE (2 classifications), Qi deficiency and blood stasis syndrome, diseased vessel (left anterior descending branch), creatinine clearance rate (CRUSADE
6 classification), and systolic blood pressure (CRUSADE 5 classification) all contributed to the occurrence of GI bleeding ( $\alpha=0.10)$ (Table 7).

5.5. Multiple Factors Regression Analysis of Risk Factors and TCM Syndromes of GI Bleeding after PCI. Multiple factors regression analysis was conducted focusing on the predictors presenting with statistical significance: age, BMI, CRUSADE score (5 classifications), CRUSADE score (2 classifications), admitting diagnosis, TCM syndromes (2 classifications), diseased vessel (LAD), heart failure signs CRUSADE score (2 classifications), creatinine clearance rate CRUSADE score (5 classifications), and systolic blood pressure (5 classifications). The regression model indicated that CRUSADE score (5 classifications) and TCM syndromes (2 classifications) were significantly correlated with GI bleeding after PCI $(\alpha=$ 0.05). 
TABLE 3: Clinical characters of GI bleeding and non-GI bleeding groups.

\begin{tabular}{|c|c|c|c|}
\hline Predictor & $\begin{array}{l}\text { GI bleeding group } \\
(n=31)\end{array}$ & $\begin{array}{l}\text { Non-GI bleeding group } \\
(n=770)\end{array}$ & $P$ value \\
\hline Age (years) & $71.39( \pm 11.24)$ & $63.74( \pm 12.07)$ & 0.0005 \\
\hline Gender & & & 0.0111 \\
\hline Male N (\%) & $14(45.16)$ & $517(67.14)$ & \\
\hline Female $N(\%)$ & $17(54.84)$ & $253(32.86)$ & \\
\hline Weight $(\mathrm{kg})$ & $68.03( \pm 10.30)$ & $71.39( \pm 11.88)$ & 0.1537 \\
\hline Height (m) & $1.66( \pm 0.08)$ & $1.67( \pm 0.08)$ & 0.5435 \\
\hline BMI & $24.53( \pm 2.81)$ & $25.47( \pm 3.49)$ & 0.1361 \\
\hline Systolic pressure (mmHg) & $123.65( \pm 22.07)$ & $132.66( \pm 22.72)$ & 0.0135 \\
\hline Heart rate $($ beat $/ \mathrm{min})$ & $76.52( \pm 10.50)$ & $75.44( \pm 14.33)$ & 0.23 \\
\hline Serum creatinine $(\mu \mathrm{mol} / \mathrm{L})$ & $197.84( \pm 167.53)$ & $105.55( \pm 105.89)$ & 0.0006 \\
\hline Creatinine clearance rate (\%) & $49.02( \pm 37.86)$ & $83.94( \pm 40.44)$ & $<0.0001$ \\
\hline Hematocrit (\%) & $38.79( \pm 7.52)$ & $39.64( \pm 5.51)$ & 0.5460 \\
\hline Hospital stay (day) & $17.06( \pm 7.92)$ & $12.45( \pm 8.80)$ & 0.0001 \\
\hline Smoking & & & 0.4812 \\
\hline Never smoking (\%) & $17(54.84)$ & $419(54.42)$ & \\
\hline Smoking $N(\%)$ & $13(41.94)$ & $341(44.29)$ & \\
\hline Previous smoking $N(\%)$ & $1(3.23)$ & $10(1.30)$ & \\
\hline \multicolumn{4}{|l|}{ Medical history } \\
\hline Hypertension & $18(58.06)$ & $497(64.55)$ & 0.4603 \\
\hline Type 2 diabetes mellitus & $11(35.48)$ & $285(37.01)$ & 0.8627 \\
\hline Lipid disorder & $5(16.13)$ & $162(21.04)$ & 0.5094 \\
\hline Chronic heart dysfunction & $2(6.45)$ & $83(10.78)$ & 0.7636 \\
\hline Chronic kidney dysfunction & $3(9.68)$ & $63(8.18)$ & 0.7358 \\
\hline Anemia & $0(0.00)$ & $16(2.08)$ & 1.0000 \\
\hline Cerebrovascular disease history & $9(29.03)$ & $160(20.78)$ & 0.2695 \\
\hline Peptic ulcer & $4(12.90)$ & $59(7.66)$ & 0.2964 \\
\hline Admitting diagnosis & & & 0.2789 \\
\hline STEMI N (\%) & $10(32.26)$ & $176(22.86)$ & \\
\hline NSTEMI N (\%) & $8(25.81)$ & $145(18.83)$ & \\
\hline UAP N (\%) & $11(35.48)$ & $331(42.99)$ & \\
\hline SAP N (\%) & $2(6.45)$ & $118(15.32)$ & \\
\hline \multicolumn{4}{|l|}{ Diseased vessel } \\
\hline Left anterior descending branch & $29(93.55)$ & $614(79.74)$ & 0.0482 \\
\hline Circumflex branch & $20(64.52)$ & $416(54.03)$ & 0.2502 \\
\hline Right coronary & $19(61.29)$ & $500(64.94)$ & 0.677 \\
\hline Left main coronary & $3(9.68)$ & $67(8.70)$ & 0.7469 \\
\hline Others & $4(12.90)$ & $76(9.87)$ & 0.5398 \\
\hline Signs of heart failure & $20(64.52)$ & $373(48.44)$ & 0.0792 \\
\hline
\end{tabular}

Odd ratio (OR) of patients with extremely high score (CRUSADE score > 50) was 22.573 (95\% CI: (2.976, 171.217)), indicating that the score is the most important predictor for GI bleeding after PCI. As for TCM syndromes, OR of non-Qi deficiency and blood stasis syndrome population was 0.300 (95\% PCI: 0.14, 0.645), which is reverse with Qi deficiency and blood stasis syndrome group, implying that Qi deficiency and blood stasis syndrome is closely correlated with GI bleeding after PCI. Data is shown in Table 8

\section{Discussion}

The major finding in the current study is that Qi deficiency and blood stasis syndrome was closely associated with postPCI bleeding, implying that this syndrome might serve as a powerful predictor of GI bleeding equally to age, gender, systolic blood pressure (SBP), and creatinine clearance (Ccr).

The current study analyzed the risk factors and clinical characteristics between patients with GI bleeding and 
TABLE 4: Comparison of CRUSADE score between GI bleeding and non-GI bleeding groups.

\begin{tabular}{|c|c|c|c|}
\hline CRUSADE score of each predictor & $\begin{array}{l}\text { GI bleeding group } \\
(n=31)\end{array}$ & $\begin{array}{l}\text { Non-GI bleeding group } \\
(n=770)\end{array}$ & $P$ value \\
\hline Gender & $4.39( \pm 4.05)$ & $2.63( \pm 3.76)$ & 0.0112 \\
\hline Type 2 diabetes mellitus & $2.13( \pm 2.92)$ & $2.22( \pm 2.90)$ & 0.8632 \\
\hline Heart rate & $1.45( \pm 1.39)$ & $1.64( \pm 2.34)$ & 0.3076 \\
\hline Signs of heart failure & $4.52( \pm 3.40)$ & $3.39( \pm 3.50)$ & 0.0795 \\
\hline Perivascular disease or stroke & $6.00( \pm 0.00)$ & $6.00( \pm 0.00)$ & 1.0000 \\
\hline Hematocrit & $2.39( \pm 3.19)$ & $1.82( \pm 2.60)$ & 0.4162 \\
\hline Creatinine clearance rate & $26.58( \pm 12.45)$ & $15.64( \pm 11.66)$ & $<0.0001$ \\
\hline Systolic blood pressure & $3.68( \pm 3.07)$ & $2.42( \pm 2.36)$ & 0.0076 \\
\hline Total CRUSADE score & $51.13( \pm 16.57)$ & $35.76( \pm 16.12)$ & $<0.0001$ \\
\hline CRUSADE 5 classification & & & $<0.0001$ \\
\hline Extremely low risk N (\%) & $1(3.23)$ & $146(18.96)$ & \\
\hline Low risk $N(\%)$ & $5(16.13)$ & $171(22.21)$ & \\
\hline Moderate risk $N(\%)$ & $1(3.23)$ & $187(24.29)$ & \\
\hline High risk $N(\%)$ & $3(9.68)$ & $108(14.03)$ & \\
\hline Extremely high risk $N(\%)$ & $21(67.74)$ & $158(20.52)$ & \\
\hline CRUSADE 2 classification & & & $<0.0001$ \\
\hline$\geq 41 N(\%)$ & $24(77.42)$ & $266(34.55)$ & \\
\hline$<41 N(\%)$ & $7(22.58)$ & $504(65.45)$ & \\
\hline
\end{tabular}

TABLE 5: Comparison of TCM syndromes between GI bleeding and non-GI bleeding groups.

\begin{tabular}{lccc}
\hline TCM syndrome & GI bleeding group $(n=31)$ & Non-GI bleeding group $(n=770)$ & $P$ value \\
\hline Heart blood stasis & $1(3.23)$ & $125(16.23)$ & 0.0627 \\
Qi deficiency and blood stasis & $19(61.29)$ & $279(36.23)$ & $46(5.97)$ \\
Qi stagnation and blood stasis & $1(3.23)$ & $94(12.21)$ & 0.0047 \\
Phlegm and blood stasis & $1(3.23)$ & $11(1.43)$ & 0.1619 \\
Cold congeal and blood stasis & $1(3.23)$ & $160(20.78)$ & 0.3794 \\
Qi and Yin deficiency & $3(9.68)$ & $27(3.51)$ & 0.1322 \\
Heart and kidney yin deficiency & $1(3.23)$ & $28(3.64)$ & 1.000 \\
Yang asthenia & $4(12.90)$ & & 0.0509 \\
\hline
\end{tabular}

TABLE 6: GI bleeding incidences in Qi deficiency and blood stasis syndrome and non-Qi deficiency and blood stasis syndrome.

\begin{tabular}{ccc}
\hline & Qi deficiency and blood stasis syndrome $N(\%)$ & Non-Qi deficiency and blood stasis syndrome $N(\%)$ \\
\hline GI bleeding & & $P$ value \\
No N (\%) & $281(93.67)$ & $489(97.60)$ \\
Yes $N(\%)$ & $19(6.33)$ & $12(2.40)$ \\
\hline
\end{tabular}

without GI bleeding. As is well known, age, gender, weight, SBP, and Ccr are important predictors in CRUSADE score assessment [9-11], which is in line with the present result. As explored before, age is an evidenced predictor of bleeding. The odds ratio (OR) of predischarge major bleeding increases by $30 \%$ as ACS patients' age increases by 10 years (OR 1.28, 95\% CI 1.21 1.37). With regard to gender, the incidence of bleeding complication in female is much higher than in male, possibly due to the smaller vessel, lighter weight, lower Ccr when at the same weight and blood creatinine, more comorbidities, and different pharmacological reaction when compared with males. In CRUSADE scoring system,
Ccr occupies the most proportion, which means kidney dysfunction is the principle risk factors for bleeding. The underlying mechanism mainly involves the metabolite pathway of antiplatelet agents such as aspirin and clopidogrel or anticoagulation drugs such as heparin through kidney. So when the glomerular filtration rate (GFR) reduced or kidney dysfunction occurs, drugs accumulate in body, leading to increased bleeding risks.

Interestingly, our current study found that Qi deficiency and blood stasis syndrome was closely associated with GI bleeding after PCI, and this syndrome was possibly equal to CRUSADE score when serving as bleeding predictors. 
TABLE 7: Single factor logistic regression analysis of risk factors and TCM syndromes.

\begin{tabular}{|c|c|c|c|c|c|c|}
\hline \multirow{2}{*}{ Variance } & \multicolumn{5}{|c|}{ Wald } & \multirow{2}{*}{$95 \% \mathrm{CI}$} \\
\hline & $\beta$ & SE & $\chi^{2}$ & $P$ & OR & \\
\hline Age & 1.4553 & 0.3712 & 15.3690 & $<0.0001$ & 4.286 & $(2.07,8.872)$ \\
\hline BMI & -0.6849 & 0.3352 & 4.1759 & 0.0410 & 0.504 & $(0.261,0.972)$ \\
\hline Gender & 0.9088 & 0.3690 & 6.0673 & 0.0138 & 2.481 & $(1.204,5.114)$ \\
\hline CRUSADE score, 5 classification, & 0.7534 & 0.1692 & 19.8193 & $<0.0001$ & 2.124 & $(1.525,2.959)$ \\
\hline CRUSADE score, 2 classifications & -1.8712 & 0.4362 & 18.4029 & $<0.0001$ & 0.154 & $(0.065,0.362)$ \\
\hline Smoking & 0.0541 & 0.3466 & 0.0244 & 0.8759 & 1.056 & $(0.535,2.082)$ \\
\hline History: hypertension & -0.2738 & 0.3717 & 0.5425 & 0.4614 & 0.761 & $(0.367,1.576)$ \\
\hline History: type 2 diabetes mellitus & -0.0662 & 0.3827 & 0.0299 & 0.8627 & 0.936 & $(0.442,1.982)$ \\
\hline History: lipid disorder & -0.3257 & 0.4962 & 0.4308 & 0.5116 & 0.722 & $(0.273,1.91)$ \\
\hline History: chronic heart dysfunction & -0.5606 & 0.7403 & 0.5736 & 0.4488 & 0.571 & $(0.134,2.436)$ \\
\hline History: chronic kidney dysfunction & 0.1844 & 0.6215 & 0.0880 & 0.7667 & 1.202 & $(0.356,4.066)$ \\
\hline History: anemia & -12.0836 & 518.6 & 0.0005 & 0.9814 & $<0.001$ & $(<0.001,>999.999)$ \\
\hline History: cerebrovascular disease & 0.4445 & 0.4055 & 1.2013 & 0.2731 & 1.560 & $(0.704,3.453)$ \\
\hline History: peptic ulcer & 0.5796 & 0.5526 & 1.1001 & 0.2942 & 1.785 & $(0.604,5.274)$ \\
\hline Qi deficiency and blood stasis syndrome & -1.0136 & 0.3763 & 7.2567 & 0.0071 & 0.363 & $(0.174,0.759)$ \\
\hline Diseased vessel, LAD & 1.3040 & 0.7366 & 3.1343 & 0.0767 & 3.684 & $(0.87,15.606)$ \\
\hline Diseased vessel, circumflex branch & 0.4364 & 0.3823 & 1.3035 & 0.2536 & 1.547 & $(0.731,3.273)$ \\
\hline Diseased vessel, right coronary & -0.1567 & 0.3764 & 0.1732 & 0.6772 & 0.855 & $(0.409,1.788)$ \\
\hline Diseased vessel, left main coronary & 0.1171 & 0.6208 & 0.0356 & 0.8504 & 1.124 & $(0.333,3.796)$ \\
\hline Diseased vessel, others & 0.3028 & 0.5491 & 0.3040 & 0.5814 & 1.354 & $(0.461,3.971)$ \\
\hline Number of stent implantation & 0.1579 & 0.1337 & 1.3943 & 0.2377 & 1.171 & $(0.901,1.522)$ \\
\hline Heart rate CRUSADE score & 0.0122 & 0.1509 & 0.0065 & 0.9358 & 1.012 & $(0.753,1.361)$ \\
\hline Heart failure signs CRUSADE score & 0.6602 & 0.3822 & 2.9831 & 0.0841 & 1.935 & $(0.915,4.093)$ \\
\hline Hematocrit CRUSADE score & 0.1533 & 0.1379 & 1.2349 & 0.2665 & 1.166 & $(0.89,1.527)$ \\
\hline Creatinine clearance rate CRUSADE score & 0.6728 & 0.1341 & 25.1778 & $<0.0001$ & 1.960 & $(1.507,2.549)$ \\
\hline Systolic blood pressure CRUSADE score & 0.3810 & 0.1383 & 7.5902 & 0.0059 & 1.464 & $(1.116,1.92)$ \\
\hline
\end{tabular}

TABLE 8: Multiple factors logistic regression analysis of risk factors and TCM syndromes.

\begin{tabular}{lcccccc}
\hline Variance & \multicolumn{3}{c}{ Wald } & & OR & \\
\hline Intercept & $B$ & SE & $\chi^{2}$ & $P$ & \\
CRUSADE score (5 classifications) & -3.2756 & 1.1224 & 8.5163 & 0.0035 & & \\
$\quad$ & & & & & & \\
$\quad$ Extremely high risk (>50) & 3.1168 & 1.0338 & 9.0896 & 0.0026 & 22.573 & $(2.976,171.217)$ \\
$\quad$ High risk (41 50) & 1.6601 & 1.1673 & 2.0226 & 0.1550 & 5.260 & $(0.534,51.835)$ \\
$\quad$ Moderate risk (31 40) & -0.2112 & 1.4200 & 0.0221 & 0.8818 & 0.810 & $(0.05,13.091)$ \\
$\quad$ Low risk (21 30) & 1.4968 & 1.1033 & 1.8406 & 0.1749 & 4.467 & $(0.514,38.826)$ \\
TCM syndrome, 2 classifications & -1.2031 & 0.3902 & 9.5067 & 0.0020 & 0.300 & $(0.14,0.645)$ \\
\hline
\end{tabular}

6.1. Relation between $Q i$ and Blood in TCM. According to TCM basic theory, the action of Qi on blood (including the controlling and leading action of Qi on blood) depends largely on governing function of spleen on blood. To be specific, when moving and generating function of spleen are vigor, both Qi and blood are simple, and thus the controlling ability of Qi is strengthened, enabling it to lead blood moving properly without oozing from vessels; conversely, if spleen is dysfunctional, source is insufficient, deficient Qi fails to control blood, leading to blood oozing out from vessels which is in other words, bleeding. Therefore, the major causation of Qi unable to control blood is dysfunction of spleen in moving and generating and incapability to lead blood. Qi failing to control blood induces blood oozing from vessel; this is blood stasis evoked by bleeding.

On other side, subsequent to bleeding, blood out of vessel together with blood despite within vessel but badly disturbed, impedes generation of new blood and induces bleeding once again; this is bleeding evoked by blood stasis, and vicious circle is initiated thereafter. The two processes always occur simultaneously and present as reciprocal causation in clinical settings. Actually, experimental researches have also reported the underlying molecular mechanism of this correlation between Qi and blood [12, 13]. 
6.2. TCM Theoretical Support for Qi Deficiency and Blood Stasis Syndrome as Post-PCI GI Bleeding Predictor. As investigators reported before, most patients with CHD presented palpitation, chest tightness, debilitation, preference to sighing, and other symptoms of Qi deficiency $[14,15]$ as well as blood stasis [16], either due to excessive fatigue, energy Qi consumption or feebleness with age, organ degradation, or greasy diet, spleen unable to moving and generating, moisture aggregating to phlegm, or anxiety impairing spleen inducing spleen Qi deficiency and unable to control blood. The major manifestation in clinical setting is GI bleeding such as haematemesis or melena, which is corresponding to TCM description of "deficient spleen unable to control blood and induce bleeding, and bleeding deteriorate spleen asthenia." In addition, patient with CHD needs to lie in bed for long term to get rehabilitation after PCI operation, especially those after acute myocardial infarction attack. Lack of exercise induces less food intake, thus abatement of GI movement, dysfunction of spleen in moving and generating, reduction of absorption of essence of water and grain, and finally deficiency in Qi and blood. Simultaneously, patients always present anxiety before PCI operation, as well as depression or irritation after PCI. Heart controlled mental activities are disturbed thereafter and thus organs are jeopardized leading to deterioration of Qi deficiency.

6.3. Modern Investigation of the Relation between Qi Deficiency and Blood Stasis Syndrome and GI Bleeding. Many researches have been conducted to elucidate the correlation between spleen function in TCM and circulation parameters. Chaoming Zhang demonstrated that PAIgG, PAIgM, and PAIgA3 were increased significantly in purpura patients with dysfunction of spleen in governing blood, with the most evident increase in PAIgG [17], indicating that the syndrome of spleen unable to govern blood is correlated with function of platelet. Zhang et al. reported that the rosettes of $\mathrm{C}_{3} \mathrm{~b}$ antibody in erythrocyte were the lowest in patients with syndrome of spleen unable to govern blood, implying that the immunological condition of erythrocyte was related to energy Qi in body [18]. Chen et al. demonstrated the bleeding tendency in rat with spleen Qi deficiency syndrome, and supplementing Qi and hemostasis preparation showed ideal remedial effect [19]. Wu et al. demonstrated the bleeding tendency in Qi deficiency syndrome [20], while other investigator showed the curative effect of supplementing Qi TCM formula [21].

Subsequent to the above basic findings, further investigations dedicated to illuminate the association between GI bleeding, circulation, and TCM syndromes were also reported. Fu and Jin showed that hemorheology and microcirculation abnormality could serve as the objective evidence of blood stasis syndrome, as score of erythrocyte sedimentation rate, hematocrit, plasma viscosity, blood viscosity, fibrinogen, and other microcirculation related parameters were much higher in GI bleeding group; besides, prolonged erythrocyte electrophoresis time, reduced microcirculation velocity, exudation, and bleeding were also reported, indicating that GI bleeding was closely involved with blood stasis [22]. Yonghua Shi documented the close association between blood stasis and hemorheological parameters; blood stasis acted as both result and causation of ulcer bleeding. Some activating-blood herbs could increase viscosity and hematocrit and decrease erythrocyte sediment rate, which is dual-direction regulation, which is in line with our finding.

6.4. Clinical Benefit. Occurrence of GI bleeding necessarily influences the overall clinical efficacy after PCI. Withdrawn or cessation of antithrombotic treatment, or even blood transfusion treatment, together with increased risks of major cardiovascular event undoubtedly prolongs hospital stays, with the increase of economic cost. In our study, patients presenting GI bleeding stayed in hospital for longer period than without bleeding, which is consistent with previous report $[23,24]$. Our finding indicated that TCM syndrome differentiation might serve as a potential supplement to the current predicting and scoring system of bleeding such as CRUSADE and thus promote the endeavor in clinical settings to prevent GI bleeding after PCI. In other words, for CHD patients with this syndrome, more attention and necessary intervention should be given immediately to prevent bleeding when administering dual-antiplatelet drugs after PCI in order to obtain optimal clinical benefit.

\section{Additional Points}

Limitation. Considering the importance of bleeding definition and optimization of the clinical trial design, the current investigation applied BARC bleeding score instead of previous TIMI and GUSTO score. As the result showed, of all bleeding population, BARC 1 classification had 61 cases (61.62\%); BARC 2 were 25 (25.25\%); BARC 3 were 13 (13.13\%). Considering the fact that TCM researches about PCI rarely use BARC score, the current data might serve as a good supplement.

\section{Disclosure}

Chenhao Zhang and Chaolian Huang are co-first authors.

\section{Conflicts of Interest}

The authors declare that there are no conflicts of interest in this paper.

\section{Authors' Contributions}

Professor Jie Wang has made substantial contributions for the study design and article revision. Associate Professor Chenhao Zhang was responsible for the study design, data analyzing, and article drafting. Professor Chaolian Huang had made substantial contribution for the study design and research conduction. Mingming Wang has made substantial contributions for the data analysis and article drafting. Xiaolin Kong and Guannan Liu were responsible for patient enrolling and data analyzing. 


\section{Acknowledgments}

The present research is granted by "Capital Featured Projects" of Beijing Municipal Science and Technology Commission (no. Z151100004015085).

\section{References}

[1] Cardiology CSo, "Guideline for diagnosis and treatment of patients with ST-elevation myocardial infarction," Chinese Journal of Cardiology, vol. 8, no. 38, pp. 675-687, 2010.

[2] Cardiology CSo, "Guideline for diagnosis and treatment of patients with non-ST-segment elevation acute coronary syndrome," Chinese Journal of Cardiology, vol. 40, no. 5, pp. 295303, 2012.

[3] H. Qiu, J. Chen, Y. Yang et al., "Clinical management for inhospital gastrointestinal bleeding in patients after percutaneous coronary intervention," Chinese Circulation Journal, no. 4, pp. 250-253, 2013.

[4] Administration SFaD, Guiding Principle of Clinical Research on New Drugs of TCM, China Medical Science and Technology Press, Beijing, China, 2002.

[5] M. Wu and X. Wang, Internal Medicine of Traditional Chinese Medicine, China Press of Trditional Chinese Medicine, Beijing, China, 2012.

[6] F. G. Kushner, M. Hand, S. C. Smith Jr. et al., "2009 focused updates: ACC/AHA guidelines for the management of patients with ST-Elevation myocardial infarction (updating the 2004 guideline and 2007 focused update) and ACC/AHA/SCAI guidelines on percutaneous coronary intervention (updating the 2005 guideline and 2007 focused update): a report of the American College of Cardiology Foundation/American Heart Association Task Force on Practice Guidelines," Circulation, vol. 120, no. 22, pp. 2271-2306, 2008.

[7] S. Subherwal, R. G. Bach, A. Y. Chen et al., "Baseline risk of major bleeding in non-sT-segment- elevation myocardial infarction the CRUSADE (can rapid risk stratification of unstable angina patients suppress ADverse outcomes with early implementation of the ACC/AHA guidelines) bleeding score," Circulation, vol. 119, no. 14, pp. 1873-1882, 2009.

[8] R. Mehran, S. V. Rao, D. L. Bhatt et al., "Standardized bleeding definitions for cardiovascular clinical trials: a consensus report from the bleeding academic research consortium," Circulation, vol. 123, no. 23, pp. 2736-2747, 2011.

[9] B. Ibanez, S. James, S. Agewall et al., "2017 ESC guidelines for the management of acute myocardial infarction in patients presenting with ST-segment elevation: the task force for the management of acute myocardial infarction in patients presenting with ST-segment elevation of the European Society of Cardiology (ESC)," European Heart Journal, vol. 39, no. 2, pp. 119-177, 2018.

[10] P. T. O’Gara, F. G. Kushner, and D. D. Ascheim, “2013 ACCF/ AHA guideline for the management of ST-elevation myocardial infarction: executive summary: a report of the American College of Cardiology Foundation/American Heart Association Task Force on practice guidelines," Circulation, vol. 127, no. 4, pp. 529-555, 2013.

[11] E. Abu-Assi, J. M. Gracía-Acuña, I. Ferreira-González, C. PeñaGil, P. Gayoso-Diz, and J. R. González-Juanatey, "Evaluating the performance of the can rapid risk stratification of unstable angina patients suppress adverse outcomes with early implementation of the ACC/AHA les (CRUSADE) bleeding score in a contemporary Spanish cohort of patients with non-ST-Segment elevation acute myocardial infarction," Circulation, vol. 121, no. 22, pp. 2419-2426, 2010.

[12] W.-W. Li, H. Guo, and X.-M. Wang, "Relationship between endogenous hydrogen sulfide and blood stasis syndrome based on the Qi-blood theory of chinese medicine," Chinese Journal of Integrative Medicine, vol. 19, no. 9, pp. 701-705, 2013.

[13] Y. Wang, W.-J. Chuo, C. Li et al., "Energy metabolism disorder and myocardial injury in chronic myocardial ischemia with Qi deficiency and blood stasis syndrome based on 2-DE proteomics," Chinese Journal of Integrative Medicine, vol. 19, no. 8, pp. 616-620, 2013.

[14] Y. Zhong, X. Lin, and M. Zhang, "Experience of Professor Tietao Deng to treat coronary heart disease with heart and spleen theory," Journal of New Chinese Medicine, vol. 39, no. 4, pp. 5-6, 2007.

[15] B. J. Chen, Z. Q. Pan, and X. X. Su, "Study on changes of TCM syndrome in patients with coronary heart disease before and after intervention treatment," Zhongguo Zhong Xi Yi Jie He Za Zhi, vol. 27, no. 8, pp. 689-691, 2007.

[16] T.-Y. Choi, J. H. Jun, J. A. Lee et al., "Expert opinions on the concept of blood stasis in China: an interview study," Chinese Journal of Integrative Medicine, vol. 22, no. 11, pp. 823-831, 2016.

[17] C. Zhang, B. Guo, and M. Yang, "Experimental research on platelet related antibodies in purpura patients with spleen unable to govern blood syndrome," Journal of Emergency in Traditional Chinese Medicine, no. 3, pp. 35-37.

[18] Y. Zhang, M. Yang, and X. Huang, "Measurement of erythrocyte immunological adhesion in spleen unable to govern blood syndrome-measurement data of 164 clinical cases and 65 rats," Shanghai Journal of Traditional Chinese Medicine, vol. 39, no. 11, pp. 7-9, 1993.

[19] Y. Chen, J. Chen, and S. Ji, "Study on relationship between insufficiency of spleen-Qi and bleeding factor in Pibu Tongxue Zheng," Research of Traditional Chinese Medicine, no. 1, pp. 3840, 2001.

[20] J. Wu, X.-Y. Wang, and H.-Y. Sun, "Study on the correlation between Chinese medicine syndrome and the intestinal mucosal manifestations of 137 patients with active ulcerative colitis," Zhongguo Zhong Xi Yi Jie He Za Zhi Zhongguo Zhongxiyi Jiehe Zazhi, vol. 32, no. 4, pp. 445-449, 2012.

[21] Y. T. Chu, X. Y. Zhu, Y. Y. Zhang et al., "Hemostatic effect of spleen-invigorating, Qi-replenishing and blood-containing formula on simvastatin-induced zebrafish hemorrhage model," Zhongguo Shi Yan Xue Ye Xue Za Zhi, vol. 25, no. 3, pp. 853-859, 2017.

[22] Z. Fu and Y. Jin, "Blood stasis pathological research of acute upper digestive tract bleeding," Journal of Emergency in Traditional Chinese Medicine, no. 6, pp. 254-256, 1994.

[23] X. Wan, J. Zhang, Z. Zhang, X. Lin, and X. Yang, "Effect of the preventive drugs on gastrointestinal bleeding in patients with acute myocardial infarction in coronary care unit and the cost," Chinese General Practice, vol. 13, no. 9, pp. 955-957, 2010.

[24] C.-M. Chen, H.-C. Hsu, Y.-W. Chuang, C.-H. Chang, C.-H. Lin, and C.-Z. Hong, "Study on factors affecting the occurrence of upper gastrointestinal bleeding in elderly acute stroke patients undergoing rehabilitation," The Journal of Nutrition, Health of Aging, vol. 15, no. 8, pp. 632-636, 2011. 


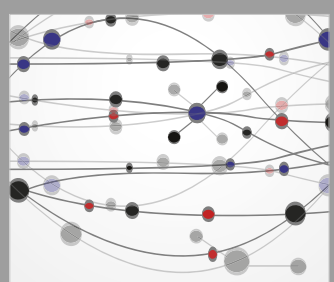

The Scientific World Journal
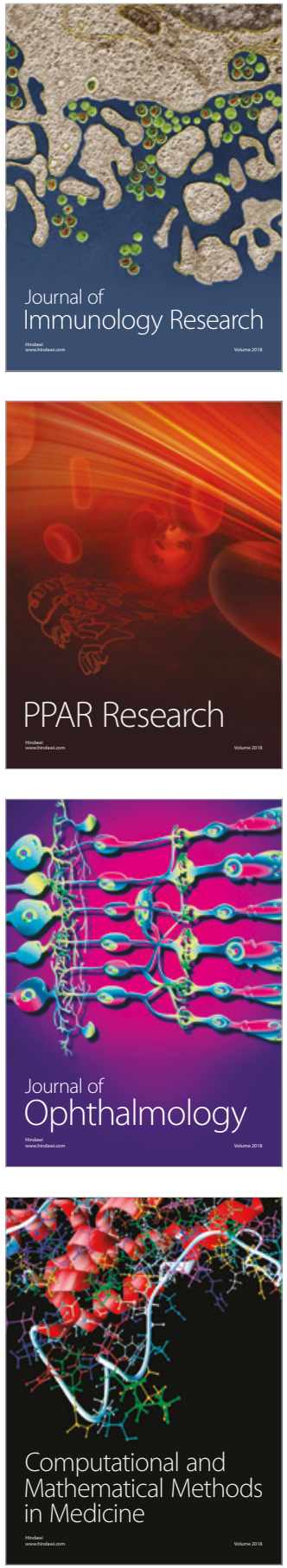

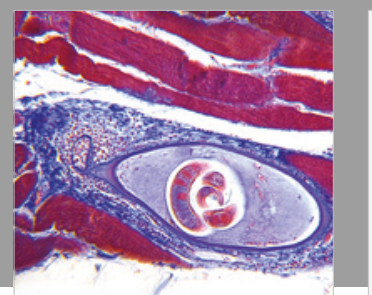

Gastroenterology Research and Practice

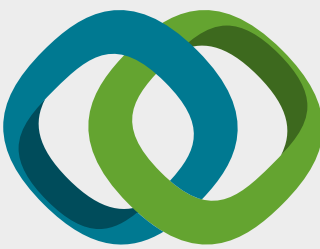

\section{Hindawi}

Submit your manuscripts at

www.hindawi.com
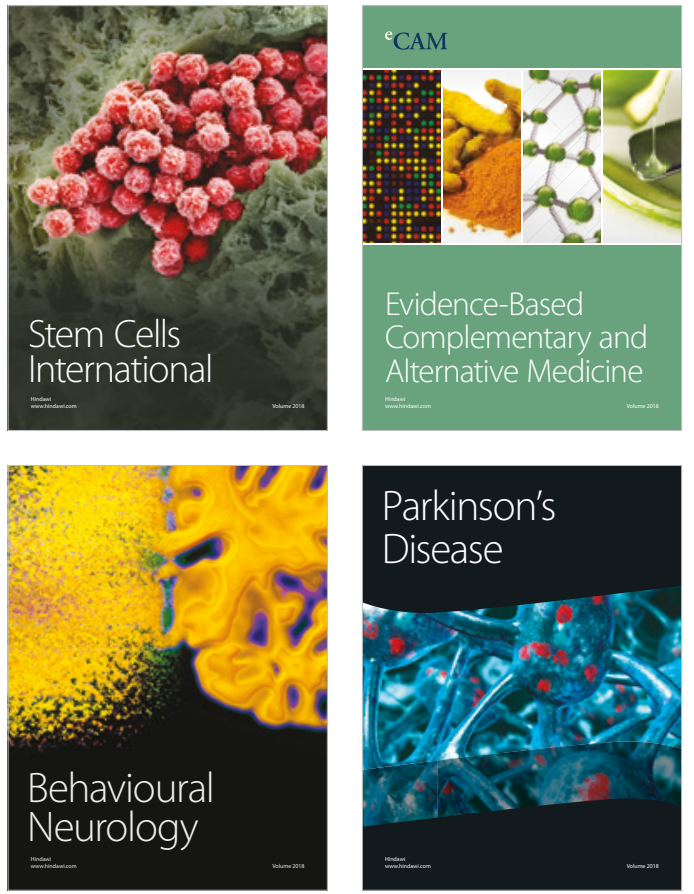

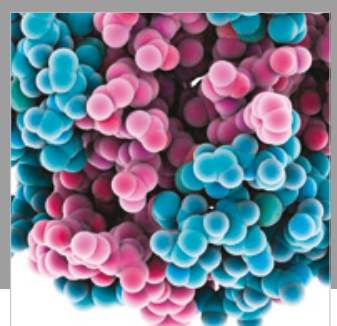

ournal of

Diabetes Research

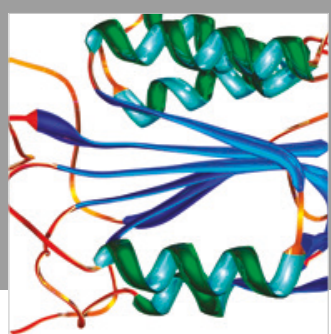

Disease Markers
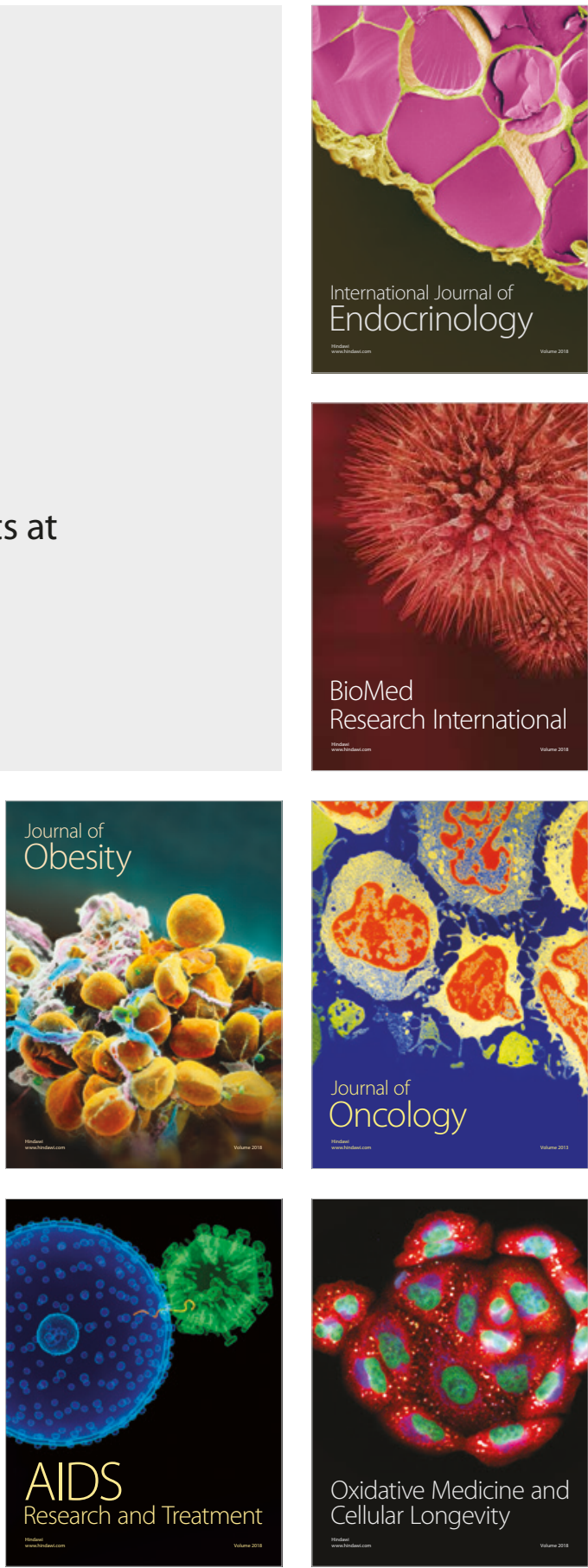